\title{
La observación sistemática participante en el hogar, una nueva técnica para el diagnóstico en la intervención con familias
}

\author{
Systematic participant observation at home. A new technique \\ for diagnosis in family \\ Orlanda DÍAz GARCíA \\ Universidad de Castilla-La Mancha \\ orlanda.diaz@uclm.es
}

Recibido: 01/08/2013

Revisado: $29 / 08 / 2013$

Aceptado: 30/07/2014

Disponible on line: 10/12/2014

\begin{abstract}
Resumen
La presente investigación presenta una nueva técnica de intervención con familias, con el objetivo de conocer su eficacia. Como indica el título, la nueva técnica- la observación sistemática participante en el hogar, pretende realizar un diagnóstico precoz y preciso. Se explicita el compendio de elementos que se requieren para su adecuada utilización: un soporte instrumental (el registro), una estrategia y un entrenamiento. El método de investigación utilizado es la estrategia del estudio de caso. A partir de la intervención con 150 familias, los 10 casos seleccionados responden a los criterios de variedad en la composición de la unidad familiar y en la problemática presentada. En cada caso se realizan dos diagnósticos a partir de sendos estudios, idénticos excepto en la sustitución de la entrevista domiciliaria por ficha técnica de observación. Los resultados obtenidos aconsejan su utilización, por encontrarse grandes diferencias cualitativas.

Palabras clave: intervención con familias, observación participante, sistematicidad y diagnóstico.
\end{abstract}

\begin{abstract}
This research presents a new technique of intervention with families in order to determine its efficiency. As the title suggests, the new technically systematic participant observation at home, intends to make an early and accurate diagnosis. It explains the elements required for the proper use: instrumental support (Register), strategy and training. The research method used is the case study approach. From the intervention with 150 families, the selected 10 cases meet the criteria of variety in the composition of the family unit and the problems presented. In each case two diagnoses was made from identical studies, except for the substitution of at home interview for datasheet observation. The results recommend its utility, because the large qualitative differences
\end{abstract}

Keywords: family intervention, participant observation, systematic and diagnosis.

Referencia normalizada: Díaz García, O. (2014): «La observación sistemática participante en el hogar, una nueva técnica para el diagnóstico en la intervención con familias». Cuadernos de Trabajo Social, 27(2): 439-452.

Sumario: Introducción. 1. Elaboración de categorías. 2. Delimitación del universo y selección de la muestra. 3. Criterios de control de calidad. 4. Resultados. 5. Discusión. 6. Referencias bibliográficas.

\section{Introducción}

La intervención con familias, que ha servido de base para la investigación, se apoya en el modelo sistémico que permite una aproximación a las familias conceptuadas como sistemas abiertos, cuya interacción determina la funcionalidad o disfuncionalidad de las relaciones familiares (Campanini y Luppi, 1991; Arruabarrena y
Paúl, 2012). Partimos de que todas las familias, ya sean las llamadas socialmente «normalizadas», o las valoradas como «con problematicidad» o «multiproblemáticas» (Cancrini, 1995; Escartín, 2004), «desorganizadas» (Minuchin y Fischman, 1998), etc., tienden a la homeostasis (Fishman, 1993; Jackson, 1957, 2009). Además, su análisis holístico examina las propiedades del 
sistema familiar como un todo (Musitu, Buelga y Lila, 1994). Este modelo nos permite acercarnos con una perspectiva terapéutica, de fortalecimiento y promoción de las potencialidades de la familia y sus miembros (Gracia y Musitu, 2000; Herrero, Estévez y Musitu, 2005).

El objeto de estudio que nos ocupa es la observación sistemática participante en el hogar, como nueva técnica para el diagnóstico en la intervención con familias. Aplicando las técnicas habituales de recogida de información y sustituyendo la entrevista domiciliaria (visita domiciliaria) por esta técnica también domiciliaria, lo que se pretende es alcanzar una mayor precisión en el diagnóstico precoz. Si se consigue, se eleva la calidad de vida de las familias, favorece la prevención de la exclusión social, obstaculiza la cronicidad de estas situaciones y ayuda a erradicar sufrimientos. Se trata de una investigación cualitativa, que estudia el objeto dentro del marco referencial y cultural de las personas a las que afecta (Taylor y Bogdan, 2000). Por otra parte, la posición del investigador como promotor de cambio (ya que está interviniendo con la familia con objetivos de salud social familiar), requiere el enfoque del modelo de investigación-acción (Martínez, 2006).

\section{Objetivos}

El objetivo general que se persigue es conocer la eficacia de la OSPH como técnica para el Diagnóstico en la Intervención con familias. Cuyos objetivos específicos son: 1) Alcanzar un diagnóstico precoz y más preciso de las necesidades y/o problemas familiares, y 2) Reducir el maltrato institucional.

\section{Método}

El método de investigación utilizado es el estudio de caso, que nos va a permitir la descripción exhaustiva del objeto de estudio. Es esencial la selección de los casos (Coller, 2005), para la presentación minuciosa de la observación sistemática participante en el hogar.

Las técnicas utilizadas para la recogida de información (estudio inicial) han sido las habituales: reuniones de coordinación con profesionales, entrevistas en el despacho, entrevistas en el hogar familiar (visitas domiciliarias), observaciones, recopilación documental, etc. En el segundo diagnóstico la única diferencia se encuentra es la sustitución de la entrevista en el hogar por la observación sistemática participante en el mismo.

La técnica de observación que se presenta responde a una metodología de observación participante. Las fuentes teóricas revisadas y estudiadas para este trabajo sobre esta técnica son múltiples: la metodología observacional de la Psicología (Cerezo, 2000; Simó, D’Ocon y Dolz, 2002; Trenado y Cerezo, 2004), ya que compartimos modelos teóricos de estudio e intervención; con la Antropología, para llegar a una buena descripción etnográfica (Guasch, 2002) y como método de investigación social (Taylor y Bogdan, 2000) en trabajos de campo. En éstos también, pero de la etnografía en salud (Amezcua y Carricondo, 2000). Con una perspectiva micro-etnográfica, ecológica o naturalista (Anguera, 1999; Anguera, Blanco y Losada, 2001; López y Frutos, 2011) y en el ámbito escolar (Ballestín, 2009). Experiencias e investigaciones en el área de salud, como la utilización del método de observación de bebés de Esther Bick (Botero, 2008); la observación terapéutica madre e hijo (Tizón, 1991; Tizón, Artigué, Goma, Parra y Marzari, 2004). En la bibliografía científica revisada no se han encontrado referencias similares en Trabajo Social. La autora trabaja desde 1997 en su preparación (Díaz, Corral y Pulido, 1997).

La técnica de la observación sistemática participante en el hogar se plantea como necesidad de realizar un diagnóstico precoz y acertado con objetivos terapéuticos para la familia. Como técnica de diagnóstico es valiosa porque la calidad y cantidad de la información que se obtiene no se logra con otra técnica ni en un tiempo tan reducido.

La técnica de la observación sistemática participante en el hogar requiere:

- Un instrumento: el registro de observaciones. El modelo básico de registro (que se encuentra en Anexo II), recogerá la dinámica familiar al menos durante tres días diferentes y cada día en momentos distintos; dos de ellos serán concertados.

- Un entrenamiento: El/la observador/a se prepara para la recogida de información, que resulta lo más difícil, para la no intervención (durante la aplicación de la técnica).

- Una estrategia para estar aproximadamente una hora cada día en el domicilio; estra- 
tegia que debe permitir la interrelación con todos los miembros de la unidad familiar y el mínimo desajuste posible en el sistema familiar.

Si se parte de un trabajo en equipo, como es este caso, es fácil realizar una distribución de roles respecto a la relación con la familia que facilite la aplicación de la técnica. En nuestro caso, para cada familia el equipo está constituido por un/a educador/a, una psicóloga y una trabajadora social.

Para comparar los diagnósticos se realizan dos de cada familia. La forma de realizar el estudio inicial en ambos diagnósticos es único en cada familia; las mismas técnicas (con la excepción de la que estamos estudiando); para analizar la información se utilizan los mismos protocolos y escalas de evaluación en los estudios que llevan a ambos diagnósticos para todas las familias. En los casos en que llegan ya diagnosticados, se realiza la observación sistemática participante en el hogar para el segundo diagnóstico. En el resto se elabora el primer diagnóstico con los resultados obtenidos del primer día de observaciones que, como mínimo, recoge tanta información como en la entrevista domiciliaria.

\section{Elaboración de categorías}

\subsection{Procedimiento}

La categorización se ha hecho con posterioridad al estudio e intervención con 150 familias, de las cuales, se realizó un estudio y una reflexión minuciosos tanto de la intervención profesional como de su comportamiento ante ésta y el seguimiento de su trayectoria posterior, lo que ha permitido la emergencia de categorías que se ilustran en las 10 familias que protagonizan este trabajo. Con posterioridad, como criterio de rigor metodológico, se ha utilizado el juicio de expertos para validarlas (Bakeman y Gottman, 1986; Flick, 2004).

Los indicadores de las áreas I, II y III han sido elaborados a partir de las escalas de evaluación utilizadas por el Servicio de Infancia de la Diputación de Sanidad y Bienestar Social de Zaragoza en 1990, con posterior adaptación del Servicio de Familia y Convivencia del Ayunta- miento de Talavera de la Reina (Díaz, Corral y Pulido, 1997) y actualizadas por la autora de esta investigación. Las escalas de evaluación actuales (véase Anexo I) se utilizan para el análisis de los datos de la fase de estudio en la intervención familiar. El área IV se evalúa mediante la adaptación que Ripol-Millet (2001) publicó sobre el eco-mapa de Hatman. Finalmente el área $\mathrm{V}$ recoge las patologías tratadas; aquellas diagnosticadas pero no tratadas y las detectadas por profesionales no diagnosticadas.

Los indicadores para medir cada una de las sub-áreas de las áreas I a III disponen de una valoración cualitativa y otra cuantitativa. Las valoraciones cuantitativas son 9 . Los valores de 1 a 3 representan una situación muy deficiente, de 4 al 6 deficiente y de 7 al 9 normalizada ${ }^{1}$.

Cada valoración cuantitativa lleva adscrita una o varias valoraciones cualitativas; al utilizar una valoración cualitativa significa: a) que esa expresión o frase es la que más se acerca a la descripción de la situación y b) que dicha descripción lleva acompañada la superación de los niveles valorativos que se encuentran por debajo. Esta fórmula tiene una excepción en la subárea 1.1.a. Situación económica: en ella se utilizan dos cuadros de datos con el número de miembros por unidad familiar y los ingresos por mes. Uno de los cuadros refleja los umbrales de pobreza severa y el otro de pobreza moderada; según los datos de la Encuesta de Condiciones de Vida para Castilla-La Mancha. De tal forma que, la valoración muy deficiente corresponde a los ingresos hasta el umbral de pobreza severa, con el valor cuantitativo 1; la de deficiente, implica ingresos entre el umbral de pobreza severa y el de pobreza moderada, con el valor 2; por último normalizada, se refiere ingresos por encima del umbral de pobreza moderada, valor 3 .

Las áreas y subáreas estudiadas, de las que se extraen las categorías son:

\section{I. Área de condiciones materiales}

Subárea 1: Estructura económica

1. a) Situación económica (escala de ingresos)*

1. b) Organización económica.

Normalizada, hace referencia a la situación media o superior a ésta que encontramos en Castilla-La Mancha. 
Subárea 2: Vivienda: condiciones materiales de habitabilidad y equipamiento.

Subárea 3: Alimentación familiar.

Subárea 4: Higiene familiar.

Subárea 5: Organización del hogar.

\section{II. Área de la situación educativo-laboral}

Subárea 1: Situación socio-profesional.

Subárea 2: Situación educativa/escolar.

- Nivel de estudios (mayores de 16 años que no cursan estudios).

- Situación escolar.

Estudios que cursa.

\section{III. Área de relaciones familiares}

Subárea 1: Relación madre/padre con hijos e hijas.

Subárea 2: Relación entre la pareja.

Subárea 3: Atención materna y paterna a las hijas y los hijos.

Subárea 4: Grado de aceptación del niño y la niña por la familia.

Subárea 5: Capacidad para asumir las funciones maternas/paternas.

\section{IV. Área de relaciones con el entorno social}

\section{V. Área de patologías individuales}

\subsection{Categorías}

El estudio inicial 1 y diagnóstico 1 son aquellos que se han realizado mediante la utilización de la técnica de la entrevista domiciliaria. La evaluación inicial 2 y diagnóstico 2 son el resultado del estudio y diagnóstico aplicando la técnica de la observación sistemática participante en el hogar.

A partir de las categorías utilizadas en los protocolos de intervención del Servicio de Familia y Convivencia de Talavera de la Reina (Blanco, Díaz y Pulido, 1994; Díaz, Corral y Pulido, 1997), se han seleccionado las categorías susceptibles de presentar variaciones entre el diagnóstico 1 y el 2.

Patologías individuales. Se analiza si el problema ha sido detectado, diagnosticado y tratado por otros servicios (de salud, educativos, etc.) y si se dispone de un informe.
Subcategorías:

- Físicas

- Psíquicas

- Sociales.

Conflictividad en la pareja. Todo tipo de conflictividad grave y/o frecuente entre la pareja (Sánchez, 2010).

Subcategorías:

- Maltrato unidireccional: sólo un miembro de la pareja maltrata al otro miembro física y/o psicológicamente.

- Maltrato bidireccional: hay una relación de pareja de maltrato entre ambos, sea físico y/o psicológico.

Riesgo para los/as menores. Se analiza cualquier tipo de riesgo familiar de gravedad para el desarrollo físico, psíquico y emocional del/a menor (Jiménez, Moreno y Palacios 1995; Allueva, 2011).

Subcategorías:

- Maltrato: contempla como atributos todos los tipos de maltrato infantil en la familia: maltrato físico, negligencia, maltrato psicológico o emocional, abandono, abuso sexual, explotación laboral, corrupción, incapacidad parental/marental para asumir funciones maternas/paternas, maltrato prenatal y síndrome de Munchausen por poderes.

- Vínculo emocional, que analiza dos atributos: ausencia o existencia del vínculo de apego del/la niño/a con el padre, la madre o con cualquier persona que haga alguna función maternal o paternal. Se recoge en múltiples investigaciones en las que se examina la relación entre el apego y la psicopatología y entre el apego y el maltrato (Balluerka, Lacasa, Gorostiaga, Muela y Pierrehumbert, 2011). Aunque la ausencia del vínculo de apego se puede analizar como consecuencia de algún tipo de maltrato, se va a analizar de forma independiente para ver, además, las posibilidades de promover el vínculo con la intervención profesional; ya que el vínculo no puede establecerse en cualquier momento del desarrollo, sino que tiene que producirse a tiempo (Bowlby, 1979; Fonagy, 2001).

Organización del hogar. Recoge hábitos de orden e higiene de la alimentación y del hogar 
Subcategorías:

- Organización alimentaria: horarios, previsiones, adecuación para la edad e higiene de los alimentos, etc.

- Orden y limpieza del hogar: habitaciones, enseres, ropas, etc.

Relaciones sociales. Relaciones con el entorno (salud, familia extensa, escuela, ocio, etc.)

Subcategorías:

- Conflictividad.

- Aislamiento.

\section{Delimitación del universo y selección de la muestra}

La intervención de Trabajo Social que ha permitido esta investigación se basa en el trabajo con familias a lo largo de 20 años; fruto de la intervención directa en los servicios sociales municipales (en dos municipios de Castilla-La Mancha); a la que se suma la participación en un proyecto piloto sobre Trabajo Social y salud, y a la supervisión externa de casos en la docencia universitaria. El universo lo constituyen todas las familias con menores.

El modelo teórico para la intervención es sistémico, con una conceptualización de la familia como paradigma sistémico-multiplicador (Ripol-Millet, 1993, 2000, 2001, 2011) y se sustenta en la interdisciplinariedad del equipo.

El total fueron inicialmente 150 familias con las que se ha intervenido y supervisado tanto la intervención como la evaluación, que ha llevado a una reflexión y a plantear esta investigación y de aquéllas se han seleccionado 10 familias como muestra del presente trabajo. La selección responde a los siguientes criterios: variedad en la composición de la unidad familiar y variedad en la problemática presentada. La muestra es no probabilística sino intencional, donde prima la profundidad sobre la extensión. El muestreo se ha basado en la comparación constante. Se han seleccionado algunas familias entre aquellas que, de forma suficiente, pueden contribuir a la consecución de los objetivos fijados.

\section{Criterios de control de calidad}

Para que los valores de calidad se encuentren en consonancia con el objeto de estudio, el proceso, los modelos teóricos de referencia, los resultados y conclusiones alcanzadas, cabe una míni- ma referencia epistemológica y ontológica. A nivel epistemológico, se hace hincapié en el enfoque dialéctico entre sujeto y objeto. El objeto de estudio (observación sistemática participante en el hogar) trata de conocer a un sujeto en interacción (la familia). Esta cuestión difícil teniendo en cuenta la complejidad de los hechos sociales, pero actualmente contemplada como característica de fortaleza del Trabajo Social (Vázquez, 1998; Teater, 2010).

El elemento ontológico que se reseña es que cada realidad social se conforma como una totalidad organizada con una fuerte interacción de sus componentes. Esto significa que hay que mirar ese sistema, a esa familia en su contexto: valores, creencias, intereses, actitudes, límites, etcétera, lo que exige un enfoque holístico e interdisciplinario de la realidad.

Para el análisis comparativo de cada familia, tras la aplicación de las dos técnicas diferenciadas para los respectivos diagnósticos (entrevista y observación sistemática participante en el hogar), se detallan las subáreas que resultan diferentes entre los dos diagnósticos realizados (ya que las áreas podrían resultar algo generales o poco esclarecedoras). Para ello se plasman, además de los resultados finales, los de cada una de las partes del protocolo utilizado: problemas principales, relación causal entre ellos y nivel de conciencia de la familia sobre dichos problemas. Esta disección de los diagnósticos facilita la supervisión de calidad.

Para la continua revisión de la observación sistemática participante en el hogar, en sí misma y en relación con todo el proceso, adscrito a cada familia, el equipo realiza supervisiones de su implementación cada quince días. Como garantía externa de calidad, toda la investigación (proceso y resultados) se ha sometido a auditoría externa.

\section{Resultados}

El análisis de los datos se ha realizado a mano, por tratarse de un número reducido de casos. La unidad de texto seleccionada ha sido la «frase», acorde con los indicadores cualitativos (frases, también) que se han estado manejando para analizar los datos de cada familia en el estudio inicial.

De las 10 familias analizadas, en 8 de ellas se obtienen diagnósticos con grandes diferencias entre el primero y el segundo, mientras que en las otras 2, el segundo diagnóstico no se diferencia mucho del primero, sólo aquél aporta algunos de- 
talles más, pero que no modifican la visión de la problemática encontrada en éste. En las 8 familias con el segundo diagnóstico diferente, las observaciones sistemáticas participantes en el hogar han permitido conocer el riesgo real, su nivel y forma, de los/as menores; en unos casos para detectar riesgos muy graves cuando no había ningún indicio, en otros para conocer su alcance, tipo y condiciones y en otros para prevenir el incremento del riesgo ya existente con una intervención inadecuada. En 3 de las familias seleccionadas el conocimiento del vínculo establecido con el menor era crucial para el diagnóstico. En 5 familias hemos podido conocer la existencia de maltrato de los hijos: de tipo físico, negligencia, maltrato emocional, abuso sexual. En cuanto a las relaciones de pareja, se han detectado 2 casos de maltrato físico unidireccional y 1 caso de maltrato bidireccional (en una dirección predominaba el físico y en la otra el psicológico). Se han podido detectar 3 casos de patologías individuales psíquicas. Es importante destacar que en uno de los casos, a pesar de estar siendo atendida en el Servicio de salud mental por depresión, no se había detectado el trastorno psiquiátrico hasta que se practicó la observación sistemática participante en el hogar. En 3 familias han sido determinantes estas observaciones para conocer los detalles de la organización alimentaria y la organización del hogar. En una familia se ha detectado el aislamiento social. En 4 familias se ha detectado una organización económica deficiente o muy deficiente; aunque en algunas de ellas no se considera uno de los problemas principales en el diagnóstico, dado que había otros problemas más graves que causaban esta desorganización.

En las 2 familias que presentaban una analogía entre ambos diagnósticos, las observaciones sistemáticas participantes en el hogar han contribuido a dar seguridad al diagnóstico y precisión al diseño del plan de intervención.

De las 150 familias arriba mencionadas (con anterioridad a las 10 que analizamos en este tra- bajo), aquellas en una fase de implantación muy avanzada del Plan de intervención familiar, se recogieron los siguientes datos: cuando se utilizaba la entrevista domiciliaria, durante la implementación del Plan de intervención, aparecían nuevos datos que modificaban radicalmente el diagnóstico. Sin embargo, en aquellas donde se aplicaba la observación sistemática participante en el hogar era inusual; los pequeños cambios que se iban introduciendo en las supervisiones quincenales (para la evaluación continua del caso) eran adaptaciones del Plan de intervención propias de la evolución; y aunque seguían apareciendo nuevas informaciones sobre la familia, lo habitual era que fuesen complementarias, y no contradictorias con las ya obtenidas.

\section{Discusión}

El estudio y análisis de la nueva técnica aquí presentada arroja resultados excelentes en la búsqueda de un diagnóstico preciso y precoz en el complicado ámbito de la intervención con familias. Entre las actividades realizadas normalmente con una familia para la recolección de los datos del estudio inicial, las observaciones sistemáticas participantes en el hogar suponen sólo 2 actividades adicionales; sin embargo, el beneficio puede ser decisivo para el bienestar de una familia y la protección de los/as menores. En ningún caso la utilización de este tipo de técnica observacional ha introducido algún perjuicio para la familia; incluso la intromisión profesional necesaria se produce ya con la entrevista domiciliaria.

Esta forma de observación, como todas las prácticas profesionales de lo social, requiere una utilización prudente, programada, diseñada para la especificidad de cada caso, revisada y supervisada. Los/as trabajadores/as sociales asumimos una gran responsabilidad cuando intervenimos con las familias, y no debemos permitirnos cualquier práctica sino la mejor posible, porque de lo contrario estaríamos contribuyendo al maltrato institucional.

\section{Referencias bibliográficas}

Allueva, L. (2011). Situaciones de riesgo y desamparo en la protección de menores. Indret: v Revista para el Análisis del Derecho, 4,1-25.

Anguera, M. T. (1999). Observación en deporte y conducta cinésicomotriz: apliaciones. Barcelona: Edicions de la Universitat de Barcelona.

Anguera, M. T., Blanco, A. y Losada, J. L. (2001). Diseños Observacionales, cuestión clave en el Proceso de la metodología observacional. Metodología de las Ciencias del Comportamiento, 3 (2), 135-161. 
Amezcua M. y Carricondo A. (2000). Investigación cualitativa en España. Análisis de la producción bibliográfica en salud. Index Enfermería, 28 (9), 26-34.

Arruabarrena, I. y De Paúl, J. (2012). Early intervention programs for children and families: theoretical and empirical bases supporting their social and economic efficiency. Psychosocial Intervention, 21 (2), 117-127.

Bakeman, R. y Gottman J. M. (1986). Observing Interaction. An introduction to Sequential Analisys. Nueva York: Cambrige University Press.

Ballestín, B. (2009). La observación participante en Primaria ¿Un juego de niños? DificultaDificultades y oportunidades de acceso a los mundos infantiles. AIBR,4 (2), 170-308.

Balluerka, N., Lacasa F., Gorostiaga, A., Muela, A. y Pierrehumbert, B. (2011). Versión reducida del cuestionario CaMir (CaMir-R) para la evaluación del apego. Psicothema, 23 (3), 486-494.

Blanco, A., Díaz, O. y Pulido, L. (1994). Programa de Familia y Convivencia de los Servicios Sociales Sociales de Talavera de la Reina. Revista de Servicios Sociales y Política Social, 33 (I), 59-72.

Botero, H. (2008). ¿Qué es la observación de bebés? Algunas consideraciones. Psicoanálisis XX (2), 103-124.

Bowlby, J. (1979). Vinculos afectivos: formación, desarrollo y pérdida. Madrid: Ediciones Morata.

Campanini, A.M. y Luppi, F. (1991). Servicio social y modelo sistémico. Barcelona: Paidos.

Cancrini, L. (1995). La caja de Pandora. Barcelona: Paidos.

Cerezo, M.A. (2000). Interacciones familiares: Un sistema de evaluación observacional. Valencia: Universidad de Valencia.

Coller, X. (2005). Estudio de casos: Cuadernos Metodológicos. Madrid: CIS.

Díaz, O., Corral, A.B. y Pulido, L. (1997). Importancia de la relación de pareja en la atención a los a los hijos. Intervención psicosocial, 6 (1), 127-132.

Escartín, M. J. (2004). Familias multiproblemáticas y Servicios Sociales. Boletín informativo Trabajo Social, 6.

Fishman, C. H. (1993). Intensive Structural Therapy: Treating Families in Their Social Conflict. Harpercollins (Agency Plan).

Flick, U. (2004). Introducción a la investigación cualitativa. Madrid: Morata.

Fonagy, P. (2001). Teoría del apego y psicoanálisis. Barcelona: Editorial SPAXS.

Gracia, E. y Musitu, G. (2000). Psicología social de la Familia. Barcelona: Paidós

Guasch, O. (2002). Observación Participante. Madrid: CIS.

Herrero, J., Estévez, E., y Musitu, G. (2005). El rol de la comunicación familiar y del ajuste escolar en la salud mental del adolescente. Salud Mental, 28 (4), 81-89.

Jackson, D. (1957). The Question of family homeostasi. Psychiatric Quarterly Suplement, 31, 7990.

Jackson, D. (2009). La homeostasis familiar y el médico. Cuadernos de información y comunicación, 14, 15-20.

Jiménez, J.M., Moreno, M.C. y Palacios, J. (1995). El maltrato infantil: Concepto, tipos, etiología. Infancia y Aprendizaje. Journal for the Study of Education and Development, 71, 7-22.

López, A. y Frutos, H. (2011). Cohesión grupal: un estudio observacional de su incidencia en Educación Física. Ágora Para la Educación Física y el Deporte, 13 (3), 397-410.

Martínez, M. (2006). La Investigación Cualitativa (Síntesis Conceptual). Revista IIPSI, 9 (1), 123146.

Minuchin, S. y Fischman, H. (1998). Family therapy techniques. England: Harvard University Press.

Musitu, G. Buelga, S. y Lila, M. S. (1994). Teoría de Sistemas. En: G. Musitu y P. Allat (eds.), Psicosociología de la Familia (pp. 47-79). Valencia: Albatros.

Ripol-Millet, A. (1993). La mediación familiar. Barcelona: Centre d'Estudis Jurídics i Formació Especialitzada.

Ripol-Millet, A. (2000). Mediación familiar. En: J. Navarro y J. Pereira (eds.), Parejas en situaciones especiales. Barcelona: Paidós. 
Ripol-Millet, A. (2001). Familias, Trabajo Social y Mediación. Barcelona: Paidos Ibérica.

Ripol-Millet, A. (2011). Estrategias de Mediación en Asuntos Familiares. Madrid: Reus.

Sánchez, M.L. (2010). Interacción afectiva-sexual y adaptación en conflictos de pareja. (Tesis doctoral inédita). Universidad Nacional de Educación a Distancia, España.

Simó, S., D’ Ocon, A. y Dolz, L. (2002). Comparación entre los patrones interaccionales madre-hi madre-hijo y la calidad del apego a los 15 meses de edad: un análisis de validación. Metodología de las Ciencias del Comportamiento (volumen especial), 516-519.

Taylor,S.J. y Bogdan, R.(2000). Introducción a los métodos cualitativos en investigación. La búsqueda de los significados. Barcelona: Paidós.

Teater, B. (2010). An introduction to applying Social Work Theories and Methods. Nueva York: Mc Graw Hill.

Tizón J L (1991). La Observación Terapéutica del Niño junto con su Madre en la clínica Infantil. Clínica y Análisis Grupal, 13 (3), 417-443.

Tizón, J.L., Artigué, J., Goma, M., Parra, B. y Marzari, B. (2004) Observación de bebés en uni una unidad funcional de atención a la salud mental de la primera infancia. Cuadernos de de Psiquiatría y Psicoterapia del Niño y del Adolescente, 37-38, 85-112.

Trenado, R. y Cerezo, M.A. (2004).Codificación de la Interacción Temprana Materno Infantil, CIT$M I-R$. Recuperado de: http://psicologia.ull.es/archivos/.../InteraccionTempranaMaternoInfantil.pdf.

Vázquez, O. (1998). Pensar la epistemología del trabajo social. Alternativas: Cuadernos de Trabajo Social, 6, 269-286.

ANEXO I. Escalas para medida de indicadores de estudio inicial

\section{CONDICIONES MATERIALES}

1. Escalas de estructura económica

1.a) Escala de situación económica

1. Muy deficiente: desde sin ingresos hasta el umbral de pobreza severa.
2. Deficiente; ingresos entre el umbral de la pobreza severa y el umbral de pobreza moderada.

3. Normalizada (integración): por encima del umbral de pobreza moderada.

\begin{tabular}{|l|l|l|l|}
\hline \multicolumn{2}{|c|}{ Pobreza severa } & \multicolumn{2}{c|}{ Pobreza moderada } \\
\hline $\mathbf{N}^{\circ}$ de miembros & Ingresos/mes & \multicolumn{1}{c|}{$\mathbf{N}^{\circ}$ de miembros } & Ingresos/mes \\
\hline 1 & & 1 & \\
\hline 2 & & 2 & \\
\hline 3 & & 3 & \\
\hline 4 & & 4 & \\
\hline 5 & & 5 & \\
\hline 6 & & 6 & \\
\hline 7 & & 7 & \\
\hline 8 & & 80 mas & \\
\hline 9 & & & \\
\hline 10 & & & \\
\hline 11 & & & \\
\hline 12 & & & \\
\hline
\end{tabular}


1.b) Escala de organización económica

1. Gastan el dinero de inmediato.

2. Cortes de luz, agua, etc.

3. Deudas constantes.

4. No llegan a final de mes.

5. Priorizar otras necesidades sobre las básicas.

6. Cubren algunas necesidades básicas como alimentación y vestido; pero no vivienda.

7. Cubre necesidades básicas.

8. Administración adecuada aunque falten ingresos.

9. Se invierte en el hogar. Ahorro.

Nota: Necesidades básicas: alimentación, vestido, vivienda.

\section{Escala de vivienda: condiciones materia- les de habi-tabilidad y equipamiento}

1. La vivienda se halla en mal estado estructural (faltan puertas, ventanas, techos, paredes, suelos sin pavimentar, sin luz natural, sin ventilación, con mucha humedad).

2. La vivienda carece de agua.

- Carece de luz eléctrica.

- Carece de retrete.

3. La vivienda carece de todo elemento de calefacción.

- La vivienda no tiene ducha.

- El agua y/o la luz eléctrica se hallan muy deterioradas (cables por fuera y en mal estado; agua o luz eléctrica insuficiente).

- La vivienda tiene una mala ventilación (habitaciones que no tienen ventanas al exterior).

- Hay humedad en la casa.

4. Hay hacinamiento en la vivienda:

- Cuatro niños o personas por dormitorio.

- Tres niños en un dormitorio sin literas.

- Varios niños duermen habitualmente en una cama.

5. La vivienda carece de agua caliente.

- No tiene frigorífico.

- Se emplea alguna habitación para varios usos.
6. Las puertas o ventanas de la vivienda están deterioradas. rados.

- El suelo, techos o paredes están deterio-

- La pintura está deteriorada o inexistente.

- La instalación eléctrica o de agua están deterioradas o viejas.

7. Los muebles son viejos, inadecuados o escasos.

8. La vivienda y el equipamiento tienen un nivel en conjunto normal-modesto.

9. La vivienda y el equipamiento son buenos, de cierta calidad.

\section{Escala de alimentación familiar}

1. Los niños no comen regularmente (más de un día a la semana).

2. Comen a veces alimentos en mal estado.

3. La alimentación es especialmente inadecuada para la edad, al menos para algún niño (dar a bebés comida de adultos, por ejemplo).

4. A veces, no comen o cenan (un día a la semana).

- Algún día pasan hambre. bre.

5. La dieta es, por lo general, escasa o po-

6. Existe desorganización en el horario de comidas.

- Hay falta de higiene en la alimentación o en su preparación.

7. La alimentación es normal, aunque sin variedad.

8. La alimentación es adecuada, aunque barata-normal de precio.

9. La alimentación es adecuada y de cierta calidad.

\section{Escala de higiene familiar}

1. Los niños despiden mal olor corporal y parásitos (no se bañan ni se duchan ).

2. Los niños despiden mal olor corporal, pero no tienen parásitos.

- Los niños llevan la ropa sucia de manera habitual. 
3. Los niños llevan el pelo sucio y enmarañado habitualmente.

- Los niños llevan las manos, la cara y piernas sucias habitualmente.

4. Se lavan por las mañanas, pero no se duchan nunca.

5. Los niños presentan un aspecto general descuidado (ropa con manchas, uñas sucias, sin peinar); pero se duchan alguna vez.

6. Los niños se duchan una vez a la semana. semana.

- Se cambian de ropa interior una vez por

7. El aspecto general de los niños es cuidado.

8. Se duchan dos veces por semana.

9. Los niños presentan una mayor higiene que el anterior: buen aspecto ( pelo, uñas manos) más duchas, se cepillan regularmente los dientes, cambio frecuente de ropa interior y exterior.

\section{Escala de organización del hogar}

1. El suelo de la vivienda está sin barrer ni fregar habitualmente.

- Hay basura en el suelo sin recoger con frecuen-cia.

2. Las camas suelen estar sin sábanas o los colchones en mal estado.

- La vajilla se guarda sucia o no se recoge.

3. La ropa sucia no se recoge habitualmente. sucios.

- El baño o la cocina están habitualmente

- Se encuentran mezclados los útiles del hogar, alimentos y cosas sucias.

4. El suelo se suele barrer, pero no fregar.

5. Hay platos recogidos en la cocina, sin fregar.

6. Las camas están habitualmente sin hacer.

- Existencia de ropa amontonada en los armarios o fuera de ellos.

- Los armarios o aparadores se hallan con toda clase de cosas.
7. El suelo está habitualmente limpio (barrido y fregado).

8. La cocina y el baño están habitualmente limpios.

9. Hay orden en la cocina y los armarios.

\section{SITUACION EDUCATIVO/LABORAL}

\section{Escala de situación socio-profesional}

1. Situaciones y ocupaciones marginales (prostitución, delincuencia, ocupaciones marginales, mendicidad, vagabundeo).

2. Parados crónicos (de los que pueda decirse que «nunca han trabajado» o «nunca han trabajado fuera del hogar»).

- Viven prioritariamente de prestaciones económicas de instituciones privadas (Cáritas, etc.) o públicas (Delegación Provincial de Bienestar Social).

3. Empleos esporádicos, sin continuidad.

4. Empleos esporádicos con cierta continuidad.

5. Empleo sumergido (trabaja el cabeza de familia, pero sin papeles, sin contrato).

6. El cabeza de familia está en paro, cobrando el subsidio de desempleo: realizando o no algún curso INEM.

- El cabeza de familia tiene trabajo fijo, pero discontinuo, o empleo intermitente.

- El cabeza de familia percibe una pensión contributiva.

7. El cabeza de familia tiene un empleo fijo sin cualificación.

8. El cabeza de familia tiene un empleo fijo con cualificación.

9. El cabeza de familia tiene un empleo fijo, con una categoría profesional superior a las anteriores.

\section{Escala de situación educativo/escolar}

a) Escala de situación escolar (para menores en edad escolar obligatoria)

1. Niños sin escolarizar por los padres.

2. Absentismo escolar.

3. Asistencia muy irregular.

4. Períodos sin asistir a clase.

5. Asistencia irregular (mañana y tarde). 
6. Los padres no muestran interés.

7. Los padres muestran interés.

8. Los niños realizan las tareas escolares en casa.

9. Asisten con regularidad al colegio.

b) Escala de situación educativa (anotar en línea continua)

$\rightarrow$ Para miembros que no cursan estudios:

a) No saben leer ni escribir.

b) Primaria incompleta.

c) Primaria completa.

d) Secundaria incompleta. Sin título de secundaria o graduado escolar.

e) Secundaria completa. Primer y Segundo ci-clo. Graduado escolar. ESO

f) Bachillerato/Prueba de madurez (B.U.P/.C.O.U.)

g) Técnico profesional o módulo 2 F.P 1.

h) Técnico profesional o módulo 3 F.P 2.

i) Estudios medios o superiores.

$\rightarrow$ Para miembros que cursen estudios (especificar curso y tipo de enseñanza que desarrollan).

a) Educación especial.

b) Guardería.

c) Enseñanza infantil.

d) Enseñanza primaria.

e) Enseñanza secundaria.

f) E.P.A. - Enseñanza para Adultos.

P.G.S. - Programa de Garantía Social. E.T. - Escuela Taller.

g) F.P.

Bachillerato. C.O.U.

h) Estudios medios.

i) Estudios superiores.

\section{RELACIONES FAMILIARES}

\section{Escala de relación padres-hijos}

1. Existen malos tratos psíquicos y/o físicos.

- Los padres están privados de la tutela legal o de guarda y educación. hijos.

2. Los padres no tienen relación con los

- Los hijos viven separados de sus padres, incluso los fines de semana.

3. Los padres no se preocupan de los hijos habitualmente (pasan de ellos, aún asumiendo como obligación las atenciones primarias: viven los hijos como una carga).

4. Broncas frecuentes. Agresividad frecuente.

- Muestras claras y continuadas de no aceptar a alguno de los hijos.

5. Actitud de dejadez o abulia de los padres; los niños suelen hacer lo que quieren.

- Actitud de autoritarismo de los padres; algún niño, al menos, vive la relación con su padre o madre con miedo o temor.

6. Los padres no dedican el tiempo suficiente al menos a algún niño.

7. La relación no es negativa.

8. Hay diálogo entre padres e hijos.

9. Hay diálogo y confianza.

- El clima es afectivo, positivo.

\section{Escala de relación en la pareja}

1. Hay malos tratos psíquicos y/o físicos entre la pareja.

2. Presencia de prostitución o proxenetismo.

3. Ausencias frecuentes e injustificadas de uno de los cónyuges.

- Semiabandono por parte de uno de los cónyuges.

4. Peleas y riñas con cierta frecuencia.

5. Afirmaciones de desprecio de uno a otro.

- Insultos.

- Vivir cada uno por su lado (al menos, uno de los dos).

6. Discusiones; hay un mal entendimiento.

7. La relación no es negativa.

8. La relación es de diálogo.

9. La relación entre ambos cónyuges o compañeros es de diálogo y confianza. Hay un clima afectivo, positivo. 


\section{Escala de atención paterna a los hijos}

1. Los padres han abandonado a algún niño.

- El niño no vive con ellos, ni siquiera los fines de semana.

2. Los padres dejan a los niños solos frecuentemente sin justificación.

3. No atienden regularmente las comidas de los niños.

- La higiene familiar es muy deficiente (es decir, han alcanzado un nivel de 1, 2 ó 3 en la escala de higiene familiar).

- No ven al niño todos los fines de semana (para menores institucionalizados).

4. No hay regularidad horaria en las comidas de los niños.

— Deficiente alimentación injustificada.

5. No hay regularidad horaria en el sueño de los niños (levantarse y acostarse).

6. Dejan a los niños solos frecuentemente, aun-que sea justificadamente, a la hora de levantarse, acostarse, comer o cenar.

- No hay regularidad en la asistencia a clase.

— La higiene familiar es deficiente (4, 5 ó 6 en la escala de higiene). fantil.

- El o los niños residen en un Hogar In-

7. Hay regularidad en la atención de comidas, incluso horaria.

— Hay regularidad horaria en el sueño.

8. Hay regularidad en la asistencia a clase.

9. Preocupación paterna por las tareas escolares (Ayuda a los niños en sus tareas escolares o problemas escolares; o se preocupan de que los niños hagan regularmente sus tareas).

- Los padres tienen contactos con los profesores del colegio.

\section{Escala del grado de aceptación del niño por la familia}

1. El niño está abandonado o lo ha sido en el último año al cuidado de terceras personas o instituciones, sin motivos justificados.
2. Insultos y manifestaciones de desprecio y rechazo al niño.

— Viven al niño como una carga.

3. Manifestaciones verbales al niño de que no le quieren con ellos.

- Plantean o han planteado últimamente objeciones para tener al niño con ellos.

- El niño reside en un grupo familiar.

4. Ridiculizar o menospreciar al niño en público.

5. Críticas injustificadas a la forma de ser, carácter o al comportamiento del niño.

- Actitud paterna de sobreprotección, con falta de estímulos en el niño.

6. No hay una actitud de rechazo hacia el niño, pero no hay preocupación por sus temas o problemas.

7. Se preocupan por cubrir sus necesidades físicas y escolares.

8. Se preocupan por los temas o problemas del niño.

9. Ayudan al niño a superar sus deficiencias o dificultades. Le motivan

\section{Escala de capacidad para asumir las fun- ciones paternas}

1. Presencia en ambos padres o en el único de alguna combinación de los puntos siguientes 2 y 3.

2. Grave incapacidad física, psíquica o moral de ambos padres o del único.

3. Presencia de alcoholismo o toxicomanías en ambos padres o en el único.

4. Incapacidad leve física, psíquica o moral de ambos padres o del único.

- Grave incapacidad física, psíquica o moral de uno de los padres.

- Abulia o dejadez permanente en ambos padres o en el único (los padres no se hacen respetar, los hijos hacen lo que quieren).

- Ausencia temporal de importancia de alguno de los padres o del único (hospital, centro penitenciario, temporeros).

5. Inmadurez psicológica para asumir a los hijos, al menos por parte de un progenitor. 
6. Incompatibilidad horaria de los dos padres o del único para atender a sus hijos en sus necesi-dades primarias (comida, cena, levantarse, acostarse, regularidad escuela)

7. Asunción de las tareas materiales, pero no escolares.
8. Los padres suelen corregir el comportamiento de los niños (los padres o tutores realizan tareas educativas).

9. Los padres aportan hábitos o pautas de conducta favorecedores de una buena inserción social.

ANEXO II. Registro de observaciones participantes en el hogar

\begin{tabular}{|c|c|c|c|c|}
\hline & $\begin{array}{c}\text { Fecha/hora/ } \\
\text { concertada } \\
\mathrm{S} / \mathrm{N}\end{array}$ & $\begin{array}{c}\text { Fecha/hora/ } \\
\text { conertada } \\
\mathbf{S} / \mathbf{N}\end{array}$ & $\begin{array}{c}\text { Fecha/hora/ } \\
\text { concertada } \\
\mathbf{S} / \mathbf{N}\end{array}$ & $\begin{array}{l}\text { Valoración } \\
\text { final }\end{array}$ \\
\hline \multicolumn{5}{|l|}{ Organización eeconómica } \\
\hline \multicolumn{5}{|l|}{ Dinero disponible } \\
\hline \multicolumn{5}{|l|}{ Cortes de suministro: luz, agua, gas, etc. } \\
\hline \multicolumn{5}{|l|}{ Deudas } \\
\hline \multicolumn{5}{|l|}{ Detalle compra dia } \\
\hline \multicolumn{5}{|l|}{ Gastos previstos } \\
\hline \multicolumn{5}{|l|}{ Inversiones/mejoras en el hobar: obras, enseres, etc. } \\
\hline \multicolumn{5}{|l|}{ Ahorros } \\
\hline \multicolumn{5}{|l|}{ Organización familiar } \\
\hline \multicolumn{5}{|l|}{ Horario comidas } \\
\hline \multicolumn{5}{|c|}{$\begin{array}{l}\text { Situación en el momento: comiendo, preparando comida, } \\
\text { no hay comida }\end{array}$} \\
\hline \multicolumn{5}{|l|}{ Estado de los alimentos: } \\
\hline \multicolumn{5}{|l|}{ Alimentación adecuada: por edad, salud } \\
\hline \multicolumn{5}{|l|}{ Dieta: pobre, media, abundante } \\
\hline \multicolumn{5}{|l|}{ Variedad: carencial, media, equilibrada } \\
\hline \multicolumn{5}{|l|}{ Higiene familiar } \\
\hline \multicolumn{5}{|l|}{ Olor corporal } \\
\hline \multicolumn{5}{|l|}{ Parásitos } \\
\hline \multicolumn{5}{|l|}{ Ropa: limpia, sucia, buen aspecto } \\
\hline \multicolumn{5}{|l|}{ Pelo: limpio, sucio, buen aspecto } \\
\hline \multicolumn{5}{|l|}{ Manos y cara } \\
\hline \multicolumn{5}{|l|}{ Aspecto general } \\
\hline \multicolumn{5}{|l|}{ Periodicidad lavado o ducha } \\
\hline Periodicidad cambio ropa interior en los menores & & & & \\
\hline
\end{tabular}


ANEXO II (cont.). Registro de observaciones participantes en el hogar

\begin{tabular}{|c|c|c|c|c|}
\hline & $\begin{array}{c}\text { Fecha/hora/ } \\
\text { concertada } \\
\mathbf{S} / \mathbf{N}\end{array}$ & $\begin{array}{c}\text { Fecha/hora/ } \\
\text { conertada } \\
\mathrm{S} / \mathrm{N}\end{array}$ & $\begin{array}{c}\text { Fecha/hora/ } \\
\text { concertada } \\
\text { S/N }\end{array}$ & $\begin{array}{l}\text { Valoración } \\
\text { final }\end{array}$ \\
\hline \multicolumn{5}{|l|}{ Organización del hogar } \\
\hline \multicolumn{5}{|l|}{ Basura en el suelo } \\
\hline \multicolumn{5}{|l|}{ Suelo barrido } \\
\hline \multicolumn{5}{|l|}{ Suelo fregado } \\
\hline \multicolumn{5}{|l|}{ Camas hechas } \\
\hline \multicolumn{5}{|l|}{ Ropa sucia sin recoger } \\
\hline \multicolumn{5}{|l|}{ Ropa limpia acumulada } \\
\hline \multicolumn{5}{|l|}{ Vajilla sucia acumulada } \\
\hline \multicolumn{5}{|l|}{ Útiles del hogar, alimentos y cosas sucias } \\
\hline \multicolumn{5}{|l|}{ Relaciones familiares } \\
\hline \multicolumn{5}{|l|}{ Relación padres-hijos } \\
\hline \multicolumn{5}{|l|}{ Relación pareja } \\
\hline Capacidades marentales/parentales & & & & \\
\hline
\end{tabular}

